\title{
Temperature-insensitive detection of low-concentration nanoparticles using a functionalized high- $Q$ microcavity
}

\author{
Wei-Liang Jin, ${ }^{1} \mathrm{Xu} \mathrm{Yi,}{ }^{2,3} \mathrm{Yi}^{-W e n ~ H u},{ }^{1}$ Bei-Bei Li, ${ }^{1,4}$ and Yun-Feng Xiao ${ }^{1}$ \\ 'State Key Laboratory of Mesoscopic Physics and School of Physics, Peking University, Beijing 100871, China \\ ${ }^{2}$ Department of Applied Physics, California Institute of Technology, Pasadena, California 91125, USA \\ 3e-mail: xyi@ caltech.edu \\ ${ }^{4}$ e-mail: libeibei@pku.edu.cn
}

Received 5 September 2012; revised 23 November 2012; accepted 27 November 2012; posted 29 November 2012 (Doc. ID 175531); published 4 January 2013

\begin{abstract}
The ability to detect nanoparticles in extremely dilute solutions in the presence of environmental noise is crucial for biosensing applications. In this paper we propose a scheme for detecting target nanoparticles through their scattering effects in a high- $Q$ whispering gallery microcavity. The detection signal, defined as the total linewidth broadening of the two new split modes that appear upon nanoparticle adsorption, is highly sensitive and proportional to the nanoparticle concentration. Furthermore, this new method of detection eliminates the requirement for strict temperature control and is capable of distinguishing the signal from the biorecognitions (e.g., antibodies) initially attached to the resonator and that from the target nanoparticles (e.g., antigens). () 2013 Optical Society of America
\end{abstract}

OCIS codes: $140.3948,280.4788,290.0290$.

\section{Introduction}

Over the past few years, optical microcavities capable of detecting unlabeled nanoparticles have become valuable tools in miniature-scale sensing [1-4]. Specifically, whispering gallery mode (WGM) microresonators are attracting much attention due to their ultrahigh $Q$ factors, small mode volumes, and potential for on-chip integration [5-7]. The conventional detection mechanism of WGM biosensing is to monitor the spectral shift induced by targets binding on the microcavity surface [을 $\underline{11}$. So far, this kind of detection has been demonstrated to possess ultrahigh sensitivity down to a single particle level. For example, [12] reported the label-free, real-time, optical detection of a single Influenza A virus by

$1559-128 \mathrm{X} / 13 / 020155-07 \$ 15.00 / 0$

(C) 2013 Optical Society of America observing the discrete changes in the resonance frequency of a microsphere cavity. By using a thermally stabilized reference interferometer in conjunction with an ultrahigh- $Q$ toroidal microcavity, Lu et al. detected individual nanobeads down to a radius of $12.5 \mathrm{~nm}$ [13]. Very recently, with plasmonic enhancement $[14, \overline{15}]$, Dantham et al. demonstrated the possibility to detect and size the smallest individual RNA virus, MS2 [16].

Nevertheless, the WGM resonant frequencies are quite susceptible to environmental noise such as thermal fluctuations $[17,18]$ so that the aforementioned experiments had to be implemented in temperature-stabilized laboratory environments, which limits their practical applications. Recently, another prototype scheme based on scatteringinduced mode splitting has been proposed and demonstrated both theoretically [19-26] and experimentally [27-34]. The detection signal used the 
resonance frequency splitting $(\Delta g)$ of the two new modes that appear upon nanoparticle adsorption, thus greatly weakening the dependence of the signal on temperature. However, this type of detection signal is strongly affected by the initial splitting of WGMs without the target particles, which actually is a common case in real label-free optical microcavity biosensors because the precovered biorecognitions on the microcavity surface are essential [1] and they also play the role of scatterers [35]. This issue can be settled by using the ratio of total linewidth broadening to total spectral shift as the detection signal [36], but, unfortunately, the problem of temperature dependence occurs here too. In this paper, we propose a new measurement strategy that uses the total linewidth broadening of the two split modes as the detection signal. Using this method not only removes the influence of the multiple types of molecules, such as biorecognitions, which are initially spread out over the microcavity surface, but also significantly reduces the detection noise caused by temperature variations. We use this signal to perform concentration detection in an aquatic environment and find that the sensing signal is proportional to the particle concentration. Although single nanoparticle detection can also be performed by extracting the step-like changes of the total linewidth broadening, this method focuses on particle concentration detection in aquatic environments by performing a statistical analysis of multiple particle binding events.

\section{Theoretical Model of Total Linewidth Broadening}

The detection system is a silica microtoroid coupled by a fiber taper, as shown in Fig. 1(a). Here we schematically describe the process of label-free microcavity biosensing with the mode splitting mechanism. A perfect microcavity supports a pair of counterpropagating modes (clockwise and counterclockwise modes) with the same resonant wavelength and
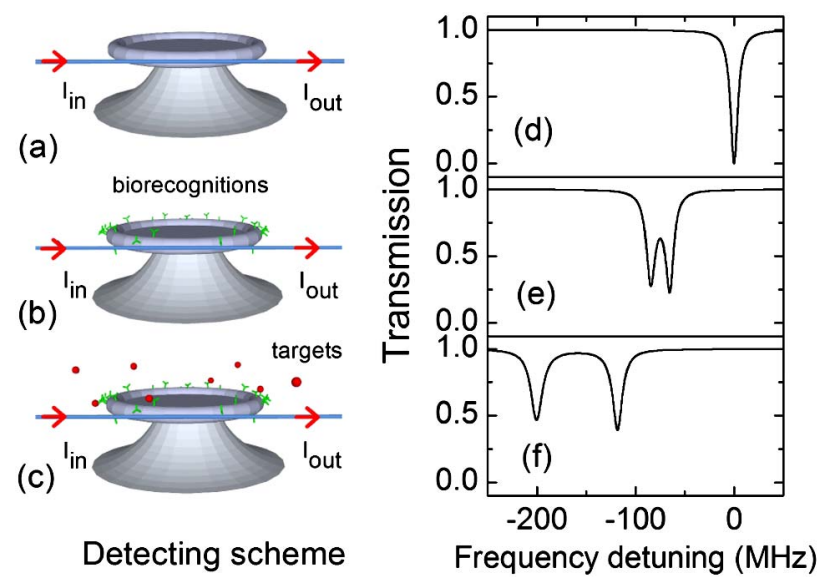

Fig. 1. (Color online) Schematic illustrations of (a) a fiber taper coupled with a bare microtoroid, (b) a microtoroid covered with the biorecognition molecules, and (c) a microtoroid covered with the biorecognition and the target molecules binding to the biorecognitions. (d)-(f) Corresponding transmission spectra of (a)-(c). damping rate [28]. Thus, the transmission spectrum of the coupling system shows a single Lorentzian dip, as depicted in Fig. 1(d). For practical uses of optical biosensing, the microcavity surface should be precovered with specific biorecognitions [Fig. 1(b)]. As a result, such degeneracy is lifted since the two travelling modes couple with each other and split into two new modes with different resonant frequencies [23]. In this case, the transmission spectrum exhibits two resonances, as shown in Fig. 1(e). Finally, target nanoparticles are specifically captured by the functionalized microcavity due to the biorecognitions [Fig. 1(c)], inducing further scattering effect, and the transmission spectrum exhibits a redistributed doublet line shape [Fig. 1(f)].

Previous experiments used the resonant frequency splitting as the detection signal [27-34], but such methods are difficult to apply to label-free optical biosensing. This is because the precovered biorecognition molecules can interact with targets through WGM fields, and this effect cannot be removed by resetting the zero point of the splitting signal [35], thus degrading the validity of the detection events. In order to illustrate the effect of the precovered biorecognition on the amount of frequency splitting, we compare the frequency splitting with and without the precovered biorecognitions. In our simulation, the microtoroid has a major (minor) diameter of $80(5) \mu \mathrm{m}$, mode function of the fundamental WGM $f(\theta)=0.45 \times \exp \left(-2.95 \theta^{2}\right) \quad(\theta$ in radian measure units) and the corresponding mode volume of $330 \mu \mathrm{m}^{3}$ in $680 \mathrm{~nm}$ band. Here we consider a special situation in which the biorecognition covers half of the cavity surface, in which case the frequency splitting induced by antibodies reaches its maximum [35]. The numerical results are displayed in Fig. 2(a), which shows the frequency splitting with (red dotted curve) and without (black solid curve) the precovered
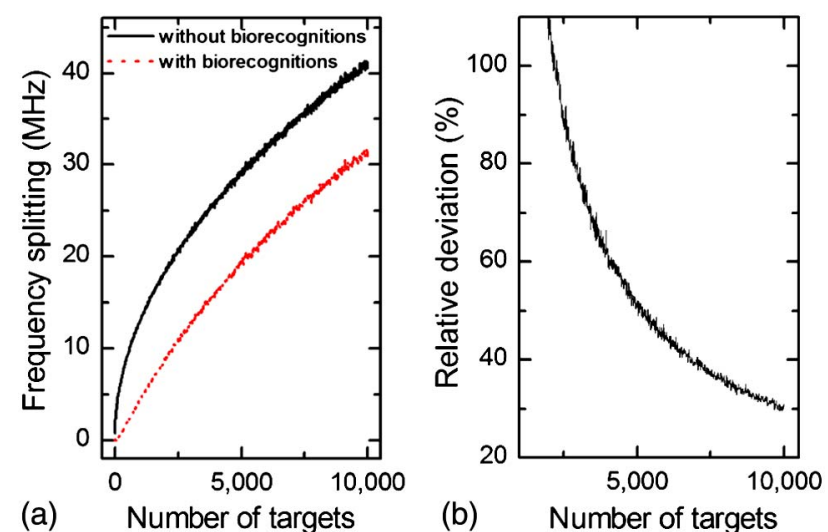

Fig. 2. (Color online) (a) Numerical frequency splittings with (red dotted curve) and without (black solid curve) precovered biorecognitions. (b) Relative deviation of the two splittings in (a). Here the biorecognition IgGs and the spherical targets with $20 \mathrm{~nm}$ radius are randomly deposited on the cavity surface. The refractive indices of the cavity, surrounding solution, precovered biorecognitions, and tested targets are chosen as 1.45, 1.33, 1.5, and 1.5, respectively. 
biorecognition when different numbers of spherical nanoparticles (radius of $20 \mathrm{~nm}$ ) are deposited. Note that both of the frequency splittings before target nanoparticles binding are set to zero. There exists significant deviation between the two curves. The deviation [Fig. 2(b)] remains larger than $30 \%$ even when the number of target molecules exceeds $1 \times 10^{4}$, which is not a favorable result for actual applications.

We now turn to exploring other sensing signals that are more appropriate for biosensing using functionalized microcavities. In mode splitting sensing mechanisms, two important parameters are used to quantitatively describe the transmission: the coupling strength between the two new modes (corresponding to the frequency splitting) and the additional linewidth (corresponding to the optical loss) [23]. The frequency splitting is affected by all the scatterers entering the cavity's evanescent field, including the biorecognition molecules and the target molecules, and the two contributions cannot be distinguished, while the contributions to the total linewidth of the two split modes, corresponding to the optical loss, can naturally be distinguished since optical loss induced by the newly arrived molecules is independent from that induced by the molecules already present. Therefore, an intuitive proposal is to use the total linewidth as the detection signal to perform biosensing. In addition, the thermal fluctuations (induced by both the temperature fluctuations of the environment and the probe laser) usually only lead to an obvious mode shift, with less of an impact on the mode linewidth. Therefore, the total linewidth is an ideal sensing signal in practical biosensing. The effect of thermal noise on the total linewidth broadening will be scrutinized in Section 4 .

Consider a fiber-microtoroid coupling system, with both $N_{b}$ identical biorecognition molecules and $N_{t}$ identical spherical scatterers both randomly adsorbed on the surface of the microtoroid. The transmission spectrum can be described by the frequency shift $g_{ \pm}=\omega_{ \pm}-\omega_{0}$ of the two new modes relative to the original mode, and the linewidth difference $\Gamma_{ \pm}=\gamma_{ \pm}-\gamma_{0}$, where $\omega_{0}\left(\omega_{ \pm}\right)$and $\gamma_{0}\left(\gamma_{ \pm}\right)$are the resonant frequencies and linewidths before (after) mode splitting ("+" and "-" denote the two split modes). By using the Weisskopf Wigner semi-QED treatment $[28,37]$, an analytical calculation can be done. We then obtain the expected values of $g_{ \pm}$and $\Gamma_{ \pm}$.

Referring to [23], both the frequency splitting $\left(g_{+}-g_{-}\right)$and the linewidth difference $\left(\Gamma_{+}-\Gamma_{-}\right)$are proportional to $N_{t}^{1 / 2}$. This nonlinearity indicates that the frequency splitting $\left(g_{+}-g_{-}\right)$and linewidth difference $\left(\Gamma_{+}-\Gamma_{-}\right)$induced by an adsorbed particle depends on the number of previously adsorbed particles. In other words, the contribution from the biorecognition molecules cannot be removed by resetting the zero point. We now analyze the total frequency shift $\Delta\left(g_{+}+g_{-}\right)$and the total linewidth broadening $\Delta\left(\Gamma_{+}+\Gamma_{-}\right)$. The results are $\Delta\left(g_{+}+\right.$ $\left.g_{-}\right)=2 \sum_{n=1}^{N_{t}} g_{n} \quad$ and $\quad \Delta\left(\Gamma_{+}+\Gamma_{-}\right)=2 \sum_{n=1}^{N_{t}} \Gamma_{n}$, respectively, with $g_{n}$ and $\Gamma_{n}$ representing the coupling strength and the scattering loss induced by the $n$th particle, respectively [35]. We see that the change in the two parameters induced by the $N$ th particle does not depend on the previous particles. However, the total frequency shift $\Delta\left(g_{+}+g_{-}\right)$as mentioned above, is strongly affected by the thermal noise, making it unusable as a detection signal, either, whereas the mode linewidth is less affected by the thermal fluctuations, making the total linewidth broadening $\Delta\left(\Gamma_{+}+\Gamma_{-}\right)$an ideal sensing signal for multiple particle detection. Total linewidth broadening can be written as

$$
\Delta\left(\Gamma_{+}+\Gamma_{-}\right)=2 \sum_{n=1}^{N_{t}} \Gamma_{n}=\frac{\alpha_{t}^{2} \omega_{0}^{4}}{3 \pi \nu^{3} V_{m}} \sum_{n=1}^{N_{t}} f^{2}\left(\theta_{n}\right),
$$

where $\alpha_{t}, f\left(\theta_{n}\right), V_{m}$, and $v$ denote the polarizability of the particles, the cavity mode function at the position of the $n$th particle, the mode volume, and the velocity of light in the surrounding medium, respectively. In Section $\underline{3}$, we will discuss the feasibility of using this sensing signal to perform concentration detection in aqueous environments.

\section{Concentration Detection with the Proposed Sensing Signal}

As we can see from Eq. (1), the proposed sensing signal strongly depends on the binding positions of the particles on the cavity surface. This is because the nanoparticles binding at different positions will bring different optical losses since the mode function $f\left(\theta_{n}\right)$ varies greatly at different positions on the cavity surface. Because of Brownian motion, the different molecules in a liquid will position themselves randomly on the cavity surface. By repeatedly performing Monte Carlo simulations, we can obtain the random positions of the target molecules on the cavity surface and find the average value and the relative standard deviation of the total linewidth change induced by the multiple target molecules. Correspondingly, we can derive the number (size) of target molecules by measuring the total linewidth broadening if we know the size (number) of the target molecules.

In our simulation, we use a microtoroid with the same size as mentioned above. In order to obtain reliable results, the Monte Carlo simulations are performed 5000 times to generate the random distributions of the multiple target molecules. Through the 5000 simulations, the average value and the relative standard deviation of the total linewidth broadening $\Delta\left(\Gamma_{+}+\Gamma_{-}\right)$with increasing particle number can be obtained, as shown in Fig. 3. In Fig. 3(a), the total linewidth broadenings induced by nanoparticles with different sizes $(r=30,40$, and $50 \mathrm{~nm}$ ) are displayed, and we can see that the sensing signal $\Delta\left(\Gamma_{+}+\Gamma_{-}\right)$shows good linear dependence on the number of targets. This implies that the proposed sensing signal is quite appropriate for 

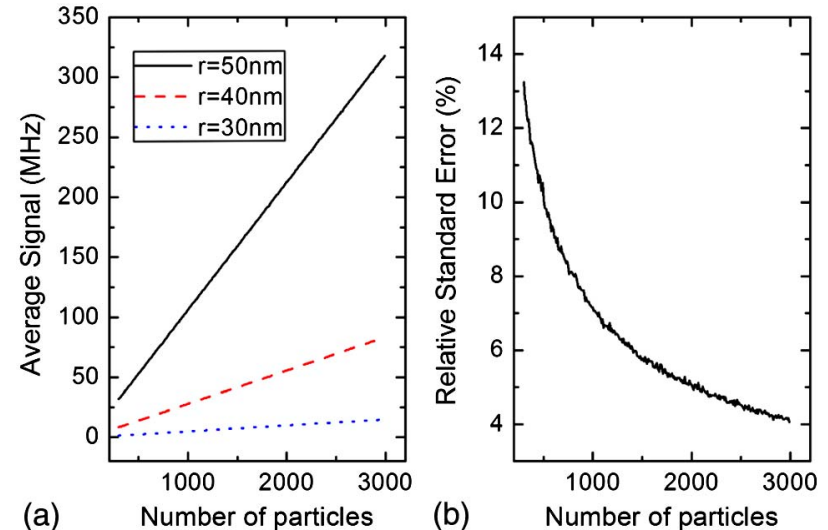

Fig. 3. (Color online) (a) Average value of the total linewidth broadening versus the number of target molecules with a radius of $50 \mathrm{~nm}$ (black solid line), $40 \mathrm{~nm}$ (red dashed line), and $30 \mathrm{~nm}$ (blue dotted line). (b) Relative standard deviation versus the number of target molecules. Note that the deviations for the three different sizes share the same curve.

concentration detection, as will be further discussed in the following. In addition, since the total linewidth broadening is proportional to $r^{6}\left(\alpha_{t}^{2}\right)$, with $r$ being the radius of the targets, the slope of the signal versus the number of targets increases greatly with an increasing target radius. Note that the mutual polarization between different target molecules is neglected here since the average distance between the target molecules is large enough [23]. From Fig. 3(b), we can see that the relative standard deviation of the sensing signal decreases rapidly with an increasing target number. For example, if a deviation smaller than $5 \%$ is necessary, about 2000 target molecules are required. This can be regarded as the detection limit. If the requirement is less rigorous, e.g., if a deviation of $10 \%$ is allowed, the detection limit is as low as 500 target molecules. This is important and beneficial for biosensing because the sensing signal is stable and reliable as long as the number of targets is large enough. In addition, the deviation is much smaller compared to that in Fig. $\underline{2}$, suggesting that the total linewidth broadening is a better sensing signal than the frequency splitting.

Since the number of target molecules can be obtained through the measurement of the total linewidth broadening, we can perform concentration measurements with the proposed sensing signal because the number of target molecules is closely related to the concentration. Here we introduce the covering ratio of target molecules $\zeta^{\prime}$, defined as $\zeta^{\prime}=$ $N_{t} / N_{0}\left(N_{0}\right.$ is the number of biological targets needed to cover the whole cavity surface), which is directly related to the concentration $C$ [38]:

$$
\zeta^{\prime}=\frac{C}{C+K_{d}},
$$

where $K_{d}$ is the dissociation constant with approximately nanomolar (nM) values for many IgGbiomolecule pairs [39]. We use $K_{d}=10 \mathrm{nM}$ as an example. Since the concentration of the target
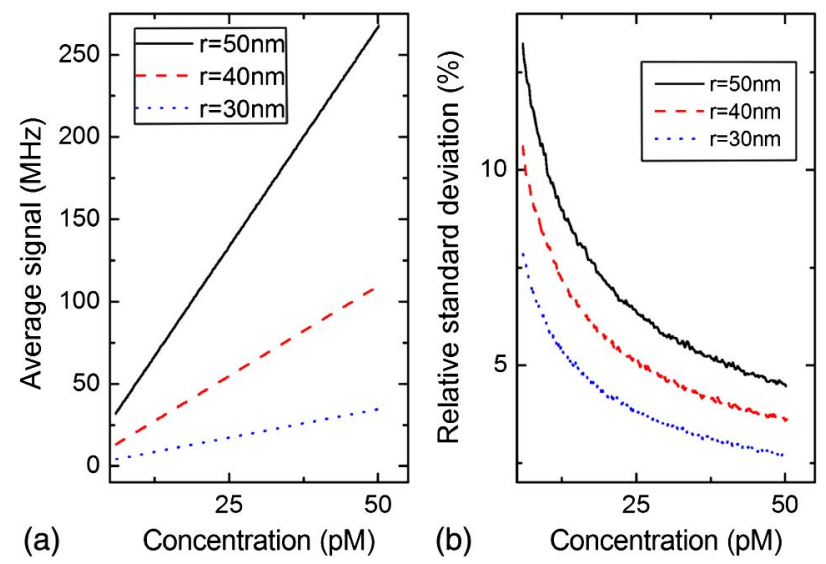

Fig. 4. (Color online) (a) Average value of the total linewidth broadening and (b) the relative standard deviation versus the concentration of target molecules with different size $r=30 \mathrm{~nm}$ (blue dotted curve), $40 \mathrm{~nm}$ (red dashed curve), and $50 \mathrm{~nm}$ (black solid curve). Here we set $K_{d}=10 \mathrm{nM}$.

molecules to be detected with this proposed signal is usually quite small, typically around $10^{2} \mathrm{pM}, \zeta^{\prime}$ can be approximated as $\zeta^{\prime}=C / K_{d}$. In this case, the relation between concentration and the number of particles on the cavity surface can be simplified as $C=K_{d} N_{t} / N_{0}$, where the concentration is proportional to the particle number $N_{t}$ for a certain particle size. As a result, the sensing signal $\Delta\left(\Gamma_{+}+\Gamma_{-}\right)$is proportional to the concentration. As depicted in Fig. 4(a), the total linewidth broadening increases linearly with concentration for nanoparticles with different sizes $(r=30,40$, and $50 \mathrm{~nm})$. Since the full covered particle $N_{0}$ is inversely proportional to $r^{2}$, the slope of the sensing signal versus particle concentration is proportional to $r^{4}$ (here we have used the aforementioned fact that the sensing signal versus particle number $N_{t}$ is proportional to $r^{6}$ ). The relative standard deviation of the total linewidth broadening versus particle concentration is displayed in Fig. 4(b). Similar to Fig. 3(b), the relative standard deviation decreases rapidly with an increasing particle concentration, and the detection limit is as low as 40 (10) $\mathrm{pM}$ for targets with a radius of $50 \mathrm{~nm}$ if a $5 \%$ (10\%) deviation is allowed. Above this limit, highaccuracy measurements could be performed. In addition, for smaller targets, the concentration measurement limit is lowered since the detection limit of the number of targets is independent of the size of the targets [Fig. 3(b)] and $N_{0}$ is inversely proportional to $r^{2}$. For example, the detection limit decreases to 14 (4) pM for targets with a radius of $30 \mathrm{~nm}$ when the allowed deviation is $5 \%(10 \%)$. Moreover, such a detection limit can be further lowered if $K_{d}$ is smaller. For example, for nanoparticles with $r=30 \mathrm{~nm}$, a detection limit of $0.14(0.04) \mathrm{pM}$ with an allowed deviation of $5 \%(10 \%)$ can be achieved for $K_{d}=0.1 \mathrm{nM}$.

We now turn to discussing the size limitations of this detection mechanism. In principle, two factors determine the particles' size limitations for this detection mechanism. The first one is the splitting 
criterion, which requires that the frequency splitting exceeds half of the total linewidth [23], and the other is the noise of the system, which limits the resolution of the measured linewidth. For the former, the splitting criterion sets an upper limit for the particle size, which is $75(95) \mathrm{nm}$ with a smaller than 5\% (10\%) deviation, as a number of large nanoparticles will induce linewidth broadenings that are larger than the frequency splitting, thus making the splitting undetectable. For the latter, the main source of noise is the linewidth of the excitation laser. For example, for the semiconductor diode laser used for the microcavity measurements in our experiment, the linewidth is about $300 \mathrm{kHz}$, which sets a lower limit for the detectable particle radius of $17(21) \mathrm{nm}$ with a smaller than 5\% (10\%) deviation. Therefore, detection of even smaller nanoparticles can be achieved by using an excitation laser with a narrower linewidth, such as a Ti:sapphire laser with a linewidth of several tens of kilohertz.

\section{Discussion on Thermal Noise}

High- $Q$ WGMs are highly sensitive to environmental disturbances. Therefore, it is essential to analyze the influence of the ambient conditions on the total linewidth broadening. One important source of noise is the thermal fluctuations induced both by the variations in the environmental temperature and by the optical power circulating inside the cavity. Typically, for a WGM with $Q=10^{8}$ the latter will bring about a temperature variation of tens of Kelvin for several milliwatts input power [40], which is much larger than that induced by the environmental noise. Consequently, in the following we concentrate on the laser-induced heating effect on the cavity and surrounding particles (the effect on water is neglected because of its large heat capacity). This thermal effect has an influence on the total linewidth broadening mainly in two aspects: (i) a change in linewidth induced by the variation in nanoparticle scattering in the cavity mode field and (ii) a change in linewidth induced by the variation of the intrinsic cavity surface defect scattering. An interesting property of this signal is that both contributions to thermal disturbance can be separated, which greatly simplifies our discussion.

(i) Linewidth change induced by the variation in nanoparticle scattering in the cavity mode field. From Eq. (1), we can see that the scattering loss is determined both by the polarizability of the scatterers $\left(\alpha_{t}\right)$ and by the cavity field [characterized by parameters $V_{m}, \omega_{0}$, and $\left.f\left(\theta_{n}\right)\right]$. When the cavity temperature changes, the refractive indices of the target molecules and the cavity will change due to the thermo-optic effect [41] (the thermal expansion effect is negligible for silica [42]). The former affects the polarizability $\alpha_{t}$ of the target molecules, and the latter will cause the mode field parameters $\left[V_{m}, \omega_{0}\right.$, and $\left.f\left(\theta_{n}\right)\right]$ to change. The contribution to temperature-induced linewidth deviation by particle polarizability and the mode field parameters are

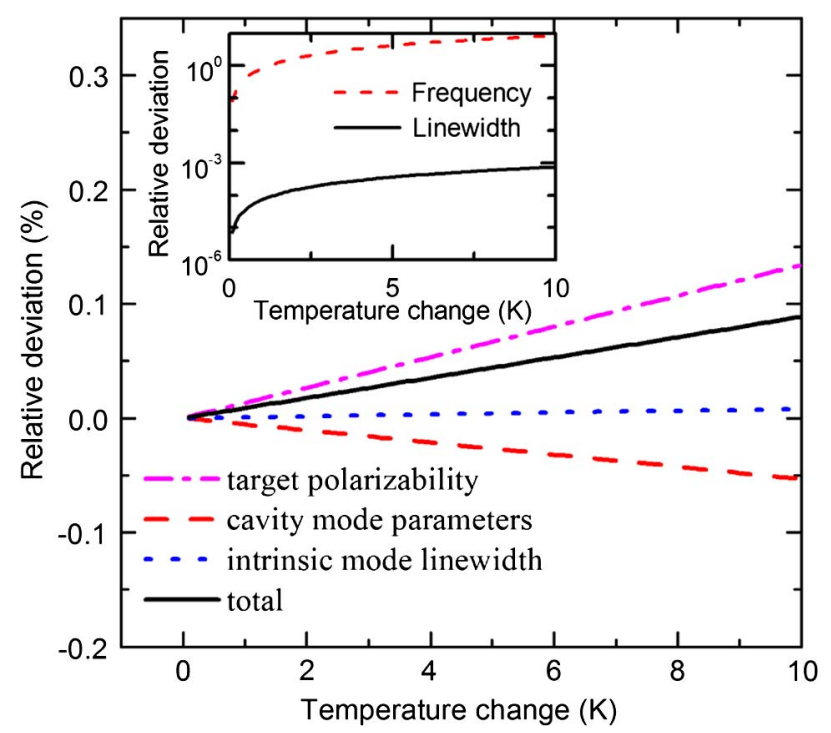

Fig. 5. (Color online) Relative deviation of total linewidth broadening versus temperature change, including the deviation induced by the variation of target particle polarizability (magenta dotteddashed line), cavity mode parameters (red dashed line), intrinsic cavity mode linewidth (blue dotted line), and their combined effects (black solid line). (Inset) Comparison of relative deviation of total frequency shift (red dashed curve) and total linewidth broadening (black solid curve) versus temperature change. Here we consider 2000 adsorbed nanoparticle targets [corresponding to the lower detection limit determined in Fig. 3(b)], with $n=1.45, d n / d T=8.55 \times 10^{-6} \mathrm{~K}^{-1}$, and $r=50 \mathrm{~nm}$.

displayed in the magenta dotted-dashed line and the red dashed line in Fig. 5, respectively, from which we can see that, with a $10 \overline{\mathrm{K}}$ temperature variation, a relative deviation of about $0.13 \%$ and $0.05 \%$ arises from the two contributions. Since the two contributions are toward the opposite directions, their total deviation is less than $0.13 \%$ with a $10 \mathrm{~K}$ temperature change.

(ii) Linewidth change induced by the variation of the intrinsic cavity surface defect scattering. For a microtoroid, the intrinsic loss (corresponding to the intrinsic linewidth) is mainly attributed to the radiation loss (caused by the curved surface), water absorption loss in the $680 \mathrm{~nm}$ band, and structural inhomogeneities (caused by surface contaminants and surface defect centers) [43]. Since our discussion focuses on ultrahigh $Q\left(\sim 1 \overline{0^{8}}\right)$ microcavities with a relatively large radius $(80 \mu \mathrm{m})$, the radiation loss is negligible. Therefore, water absorption loss and the surface inhomogeneities dominate the total loss, yet the former changes little because of the negligible thermal effect on water. For simplicity but without losing the underlying physics, for a WGM with $Q \sim 10^{8}$, the intrinsic scattering loss can be modeled by considering the inhomogeneities as a number of nanometer-sized particles deposited on the surface. With temperature fluctuations, the refractive index of these nanoparticles changes, leading to different scattering losses and correspondingly to an altered intrinsic linewidth. The deviation induced by the intrinsic mode linewidth under 
different temperature changes is shown by the blue dotted line of Fig. 5, from which we can see a less than $0.008 \%$ deviation under a $10 \mathrm{~K}$ temperature fluctuation.

Given the discussion above we see that the signal only fluctuates by $0.09 \%$ with a $10 \mathrm{~K}$ temperature change, as shown in the black solid curve in Fig. 5 . Finally, we compare the effect of temperature fluctuation on the total frequency shift and total linewidth broadening, shown in the inset of Fig. 5. It is found that the total spectral shift deviates by more than $800 \%$ under the same thermal fluctuation. Although the step-like changes in the resonant mode frequency sometimes eliminate the ambiguity of the temperature shift, it does make it experimentally difficult to distinguish the signals induced by the nanoparticle binding events from that induced by the thermal fluctuation or even the laser frequency drift. This result demonstrates that our scheme is robust even in a tough experimental environment.

\section{Conclusion}

In summary, we propose measuring the total linewidth broadening of the two split modes as a new way to detect multiple target nanoparticle binding events on a microcavity surface that has already been precovered by biorecognition molecules. The results show that the total linewidth broadening induced by the target molecules can be well separated from that induced by the precovered biorecognitions. In addition, the sensing signal increases linearly with the concentration of the targets, with a picomolar detection limit. It is also found that the total linewidth broadening is insensitive to thermal disturbance. These properties reveal that our scheme is of great potential for applications in compact label-free sensors. Finally, it should be noted that the linewidths of the two new modes strongly depend on the taper-cavity coupling strength since the coupling brings additional loss. Therefore, stable taper-cavity coupling in experiment is necessary.

B.-B. Li thanks William Clements for the helpful discussion and the paper revision. The authors acknowledge financial support from the National Natural Science Foundation of China under grant nos. 11004003, 11121091, and 11222440. Y.-F. Xiao was also supported by the Research Fund for the Doctoral Program of Higher Education (no. 20090001120004).

\section{References}

1. X. Fan, I. M. White, S. I. Shopova, H. Zhu, J. D. Suter, and Y. Sun, "Sensitive optical biosensors for unlabeled targets: a review," Anal. Chim. Acta 620, 8-26 (2008).

2. S. Blair and Y. Chen, "Resonant-enhanced evanescent-wave fluorescence biosensing with cylindrical optical cavities," Appl. Opt. 40, 570-582 (2001).

3. R. W. Boyd and J. E. Heebner, "Sensitive disk resonator photonic biosensor," Appl. Opt. 40, 5742-5747 (2001).

4. F. Vollmer and S. Arnold, "Whispering-gallery-mode biosensing: label-free detection down to single molecules," Nat. Methods 5, 591-596 (2008).
5. A. M. Armani and K. J. Vahala, "Heavy water detection using ultra-high-Q microcavities," Opt. Lett. 31, 1896-1898 (2006).

6. I. M. White, H. Oveys, and X. Fan, "Liquid-core optical ringresonator sensors," Opt. Lett. 31, 1319-1321 (2006).

7. Y.-F. Xiao, C.-L. Zou, B.-B. Li, Y. Li, C.-H. Dong, Z.-F. Han, and Q. Gong, "High-Q exterior whispering-gallery modes in a metal-coated microresonator," Phys. Rev. Lett. 105, 153902 (2010).

8. F. Vollmer, D. Braun, A. Libchaber, M. Khoshsima, I. Teraoka, and S. Arnold, "Protein detection by optical shift of a resonant microcavity," Appl. Phys. Lett. 80, 4057-4059 (2002).

9. S. Arnold, M. Khoshsima, I. Teraoka, S. Holler, and F. Vollmer, "Shift of whispering-gallery modes in microspheres by protein adsorption," Opt. Lett. 28, 272-274 (2003).

10. F. Vollmer, S. Arnold, D. Braun, I. Teraoka, and A. Libchaber, "Multiplexed DNA quantification by spectroscopic shift of two microsphere cavities," Biophys. J. 85, 1974-1979 (2003).

11. M. Noto, M. Khoshsima, D. Keng, I. Teraoka, V. Kolchenko, and S. Arnold, "Molecular weight dependence of a whispering gallery mode biosensor," Appl. Phys. Lett. 87, 223901 (2005).

12. F. Vollmer, S. Arnold, and D. Keng, "Single virus detection from the reactive shift of a whispering-gallery mode," Proc. Natl. Acad. Sci. USA 105, 20701-20704 (2008).

13. T. Lu, H. Lee, T. Chen, S. Herchak, J.-H. Kim, S. E. Fraser, R. C. Flagan, and K. Vahala, "High sensitivity nanoparticle detection using optical microcavities," Proc. Natl. Acad. Sci. USA 108, 5976-5979 (2011).

14. J. D. Swaim, J. Knittel, and W. P. Bowen, "Detection limits in whispering gallery biosensors with plasmonic enhancement," Appl. Phys. Lett. 99, 243109 (2011).

15. S. I. Shopova, R. Rajmangal, S. Holler, and S. Arnold, "Plasmonic enhancement of a whispering-gallery-mode biosensor for single nanoparticle detection," Appl. Phys. Lett. 98, 243104 (2011).

16. V. R. Dantham, S. Holler, V. Kolchenko, Z. Wan, and S. Arnold, "Taking whispering gallery-mode single virus detection and sizing to the limit," Appl. Phys. Lett. 101, 043704 (2012).

17. B.-B. Li, Q.-Y. Wang, Y.-F. Xiao, X.-F. Jiang, Y. Li, L. Xiao, and Q. Gong, "On chip, high-sensitivity thermal sensor based on high- $Q$ polydimethylsiloxane-coated microresonator," Appl. Phys. Lett. 96, 251109 (2010).

18. M. L. Gorodetsky, "Thermodynamical fluctuations in optical microspheres," Proc. SPIE 4270, 147-153 (2001).

19. K. R. Hiremath and V. N. Astratov, "Perturbations of whispering gallery modes by nanoparticles embedded in microcavities," Opt. Express 16, 5421-5436 (2008).

20. I. Teraoka and S. Arnold, "Resonance shifts of counterpropagating whispering-gallery modes: degenerate perturbation theory and application to resonator sensors with axial symmetry," J. Opt. Soc. Am. B 26, 1321-1329 (2009).

21. L. Deych and J. Rubin, "Rayleigh scattering of whispering gallery modes of microspheres due to a single dipole scatterer," Phys. Rev. A 80, 061805(R) (2009).

22. X. Yi, Y.-F. Xiao, Y. Li, Y.-C. Liu, B.-B. Li, Z.-P. Liu, and Q. Gong, "Polarization-dependent detection of cylinder nanoparticles with mode splitting in a high- $Q$ whispering-gallery microresonator," Appl. Phys. Lett. 97, 203705 (2010).

23. X. Yi, Y.-F. Xiao, Y.-C. Liu, B.-B. Li, Y.-L. Chen, Y. Li, and Q. Gong, "Multiple-Rayleigh-scatterer-induced mode splitting in a high- $Q$ whispering-gallery-mode microresonator," Phys. Rev. A 83, 023803 (2011).

24. J. Wiersig, "Structure of whispering-gallery modes in optical microdisks perturbed by nanoparticles," Phys. Rev. A 84 , 063828 (2011).

25. Y. Shen and J.-T. Shen, "Nanoparticle sensing using whispering-gallery-mode resonators: plasmonic and Rayleigh scatterers," Phys. Rev. A 85, 013801 (2012).

26. Y. Shen, D.-R. Chen, and J.-T. Shen, "statistical theory of nanoparticle sensing using a whispering-gallery-mode resonator," Phys. Rev. A 85, 063808 (2012).

27. D. S. Weiss, V. Sandoghdar, J. Hare, V. Lefêvre-Seguin, J.-M. Raimond, and S. Haroche, "Splitting of high-Q Mie modes induced by light backscattering in silica microspheres," Opt. Lett. 20, 1835-1837 (1995). 
28. A. Mazzei, S. Götzinger, L. de Souza Menezes, G. Zumofen, O. Benson, and V. Sandoghdar, "Controlled coupling of counterpropagating whispering-gallery modes by a single Rayleigh scatterer: a classical problem in a quantum optical light," Phys. Rev. Lett. 99, 173603 (2007).

29. B. Koch, Y. Yi, J.-Y. Zhang, S. Znameroski, and T. Smith, "Reflection-mode sensing using optical microresonators," Appl. Phys. Lett. 95, 201111 (2009).

30. J. Zhu, S. K. Özdemir, Y.-F. Xiao, L. Li, L. N. He, D. R. Chen, and L. Yang, "On-chip single nanoparticle detection and sizing by mode splitting in an ultrahigh- $Q$ microresonator," Nat. Photonics 4, 46-49 (2010).

31. L. He, S. K. Özdemir, J. Zhu, and L. Yang, "Ultrasensitive detection of mode splitting in active optical microcavities," Phys. Rev. A 82, 053810 (2010).

32. W. Kim, S. K. Özdemir, L. He, and L. Yang, "Observation and characterization of mode splitting in microsphere resonators in aquatic environment," Appl. Phys. Lett. 97, 071111 (2010).

33. J. Zhu, S. K. Özdemir, L. He, and L. Yang, "Controlled manipulation of mode splitting in an optical microcavity by two Rayleigh scatterers," Opt. Express 18, 23535-23543 (2010).

34. L. He, S. K. Özdemir, J. Zhu, W. Kim, and L. Yang, "Detecting single viruses and nanoparticles using whispering gallery microlasers," Nat. Nanotechnol. 6, 428-432 (2011).

35. X. Yi, Y.-F. Xiao, Y. Feng, D.-Y. Qiu, J.-Y. Fan, Y. Li, and Q. Gong, "Mode-splitting-based optical label-free biosensing with a biorecognition-covered microcavity," J. Appl. Phys. 111, $114702(2012)$.

36. J. Zhu, S. K. Özdemir, L. He, D.-R. Chen, and L. Yang, "Single virus and nanoparticle size spectrometry by whisperinggallery-mode microcavities," Opt. Express 19, 16195-16206 (2011).

37. K. Srinivasan and O. Painter, "Mode coupling and cavityquantum-dot interactions in a fiber-coupled microdisk cavity," Phys. Rev. A 75, 023814 (2007)

38. H. Zhu, I. M. White, J. D. Suter, P. S. Dale, and X. Fan, "Analysis of biomolecule detection with optofluidic ring resonator sensors," Opt. Express 15, 9139-9146 (2007).

39. S. Y. Tetin and T. L. Hazlett, "Optical spectroscopy in studies of antibody-hapten interactions," Methods 20, 341-361 (2000).

40. T. Carmon, L. Yang, and K. J. Vahala, "Dynamical thermal behavior and thermal self-stability of microcavities," Opt. Express 12, 4742-4750 (2004).

41. D. B. Levitona and B. J. Frey, "Temperature-dependent absolute refractive index measurements of synthetic fused silica," NASA Goddard Space Flight Center, Greenbelt, Maryland 20771 (2006).

42. M. L. Gorodetsky and I. S. Grudinin, "Fundamental thermal fluctuations in microspheres," J. Opt. Soc. Am. B 21, 697-705 (2004).

43. B. E. Little and J.-P. Laine, "Surface-roughness-induced contradirectional coupling in ring and disk resonators," Opt. Lett. 22, 4-6 (1997). 